and as above this is sufficient that postulates $\mathrm{I}-\mathrm{V}$ be satisfied.

With this definition of $A: B, A \supset B$ becomes the usual inclusion relation of the algebra of classes [5].

\title{
REFERENCES
}

1. E. T. Bell, Arithmetic of logic, Transactions of this Society, vol. 29 (1927), pp. $597-611$.

2. Garrett Birkoff, On combination of subalgebras, Cambridge Philosophical Society Proceedings, vol. 29 (1933), pp. 441-464.

3. R. Dedekind, Dirichlet, Vorlesungen wber Zahlentheorie.

4. E. Lasker, Zur Theorie der Moduln und Ideale, Mathematische Annalen, vol. 60 (1905), pp. 20-115.

5. Lewis and Langford, Symbolic Logic, 1932.

6. F. S. Macaulay, The Algebraic Theory of Modular Systems, Cambridge Tracts, no. $19,1916$.

7. O. Ore, Abstract Algebra I, II, Annals of Mathematics, vol. 36 (1935), pp. 406437; vol. 37 (1936), pp. 265-292.

8. B. L. van der Waerden, Moderne Algebra, vol. 2.

9. M. Ward, Some arithmetical applications of residuation, American Journal of Mathematics, vol. 59 (1937), pp. 921-926.

California Institute of Technology

\section{A NOTE ON THE MAXIMUM PRINCIPLE FOR ELLIPTIC DIFFERENTIAL EQUATIONS}

FRITZ JOHN

Let $u\left(x_{1}, \cdots, x_{n}\right)$ denote a twice continuously differentiable function of $x_{1}, \cdots, x_{n}$ in some region $R$. We write $\partial u / \partial x_{i}=u_{i}, \partial^{2} u / \partial x_{i} \partial x_{k}$ $=u_{i k}$, and occasionally $(x)$ for $\left(x_{1}, \cdots, x_{n}\right)$. A point $(c)=\left(c_{1}, \cdots, c_{n}\right)$ of $R$ may be called a proper maximum of $u$, if

$$
\begin{gathered}
u_{i}(c)=0 \quad \text { for } \quad i=1, \cdots, n, \\
\sum_{i, k} u_{i k}(c) \xi_{i} \xi_{k}<0 \quad \text { for all } \quad\left(\xi_{1}, \cdots, \xi_{n}\right) \neq(0, \cdots, 0) .
\end{gathered}
$$

A partial differential equation

$$
\sum_{i, k} a_{i k}(x) u_{i k}(x)+\sum_{i} b_{i}(x) u_{i}(x)=0
$$

(where the $a_{i k}$ and $b_{i}$ are defined in $R$ ) is called elliptic if for every $(x)$ of $R$

$$
\sum_{i, k} a_{i k}(x) \xi_{i} \xi_{k} \geqq 0
$$

for all $\left(\xi_{1}, \cdots, \xi_{n}\right)$ and $<0$ for some $\left(\xi_{1}, \cdots, \xi_{n}\right)$. 
It is well known, that a solution $u$ of (1) can not have a proper maximum.* For if $u$ had a proper maximum at $\left(c_{1}, \cdots, c_{n}\right)$, then $\sum_{i, k} a_{i k}(c) u_{i k}(c)=0$. If $A$ and $U$ denote respectively the matrices $\left(a_{i k}(c)\right)$ and $\left(u_{i k}(c)\right)$, this may be written: Trace $(A \cdot U)=0$. By a suitable orthogonal transformation $A$ may be transformed into a diagonal matrix $A^{\prime}=\left(a_{i}^{\prime} \delta_{i k}\right), U$ going over into $U^{\prime}=\left(u_{i k}^{\prime}\right)$ by the same transformation. As the trace of $A \cdot U$ is preserved, we have $\sum_{i} a_{i}{ }^{\prime} u_{i t}^{\prime}=0$; on the other hand, as $A^{\prime}$ still belongs to a semi-definite quadratic form and $U^{\prime}$ to a negative definite one, we have $a_{i}^{\prime} \geqq 0$ for $i=1, \cdots, n$, but $<0$ for some $i$, and $u_{i i}^{\prime}<0$ for all $i$. This leads to a contradiction.

A second important property of the solutions $u$ of (1) is that they form a module, that is, that every linear combination with constant coefficients is again a solution.

We shall prove that these two properties are also sufficient to characterize a family of functions as solutions of an elliptic equation (1).

TheOREM. Let $F$ be any family of twice continuously differentiable functions $u\left(x_{1}, \cdots, x_{n}\right)$ defined in $R$, such that

(a) the functions of $F$ form a module,

(b) no function of $F$ has a proper maximum.

Then there is an elliptic differential equation (1) satisfied by all functions of $F$.

Proof. Let $(c)=\left(c_{1}, \cdots, c_{n}\right)$ be a point of $R$. Let $\phi$ be the submodule of functions $u$ of $F$ for which $u_{i}(c)=0$ for $i=1, \cdots, n$. Let $Q\left(\xi_{1}, \cdots, \xi_{n}\right)$ denote the quadratic form $\sum_{i, k} u_{i k}(c) \xi_{i} \xi_{k}$ for any $u$ in $\phi$; $Q$ is certainly not negative definite. These quadratic forms form again a module $M$. Let $Q_{1}, Q_{2}, \cdots, Q_{m}$ form a basis of this module $(m \leqq n(n+1) / 2)$, such that for every $Q$ of $M$

$$
Q\left(\xi_{1}, \cdots, \xi_{n}\right)=\sum_{i=1}^{m} \lambda_{i} Q_{i}\left(\xi_{1}, \cdots, \xi_{n}\right)
$$

with certain constants $\lambda_{i}$. We know that for every $\left(\lambda_{1}, \cdots, \lambda_{m}\right)$ there are $\left(\xi_{1}, \cdots, \xi_{n}\right) \neq(0, \cdots, 0)$ such that

$$
\sum_{i=1}^{m} \lambda_{i} Q_{i}\left(\xi_{1}, \cdots, \xi_{n}\right) \geqq 0 .
$$

From this we can easily conclude that the $Q_{i}$ satisfy a linear relation

* Cf. Encyklopädie der Mathematischen Wissenschaften, vol. 2, 1.1, p. 522, or Picard, Traite d'Analyse, $3 \mathrm{~d}$ ed., vol. 2, p. 29 for the case $n=2$. The subsequent proof follows Courant-Hilbert, Methoden der Mathematischen Physik, vol. 2. 
with positive coefficients.* For let $\Sigma$ denote the set of points with coordinates $\left(Q_{1}(\xi), Q_{2}(\xi), \cdots, Q_{m}(\xi)\right)$ in an $m$-dimensional space, where $(\xi)=\left(\xi_{1}, \cdots, \xi_{n}\right)$ varies over all points of the unit sphere $\xi_{1}{ }^{2}+\xi_{2}{ }^{2}+\cdots+\xi_{n}{ }^{2}=1$. The set $\Sigma$ is closed and finite. The relation (2) may be interpreted as stating that every half-space bounded by a plane through the origin contains points of $\Sigma$. Thus the origin is contained in the convex extension of $\Sigma$. Then there exists a finite set of points $P_{1}, \cdots, P_{r}$ of $\Sigma$ and positive numbers $\mu_{1}, \cdots, \mu_{r}$, such that the origin is the center of mass of the masses $\mu_{i}$ placed at the vertices $P_{i} . \dagger$ Let $\left(\xi_{1}{ }^{i}, \cdots, \xi_{n}{ }^{i}\right)$ be the point $\left(\xi_{1}, \cdots, \xi_{n}\right)$ corresponding to $P_{i}$. Then

$$
\sum_{j=1}^{r} \mu_{j} Q_{k}\left(\xi_{1}{ }^{j}, \cdots, \xi_{n}^{j}\right)=0
$$

for $k=1, \cdots, m$, and consequently

$$
\sum_{j=1}^{r} \mu_{j} Q\left(\xi_{1}{ }^{j}, \cdots, \xi_{n}^{j}\right)=0
$$

for every $Q$ in $M$. Thus

$$
\sum_{j=1}^{r} \sum_{i, k} \mu_{j} u_{i k}\left(c_{1}, \cdots, c_{n}\right) \xi_{i}{ }^{j} \xi_{k}{ }^{j}=0
$$

for $u$ in $\phi$. Putting $\sum_{i} \mu_{j} \xi_{i}{ }^{j} \xi_{k}{ }^{j}=a_{i k}(c)$, we have

for $u$ in $\phi$. Besides

$$
\sum_{i, k} a_{i k}(c) u_{i k}(c)=0
$$

$$
\sum_{i, k} a_{i k}(c) \xi_{i} \xi_{k}=\sum_{i, j} \mu_{j}\left(\xi_{i} \xi_{i}\right)^{2} \geqq 0
$$

and $>0$ for some $\left(\xi_{1}, \cdots, \xi_{n}\right)$.

Now let $u\left(x_{1}, \cdots, x_{n}\right)$ denote an arbitrary function of $F$. The vectors $\left(y_{1}, \cdots, y_{n}\right)=\left(u_{1}(c), u_{2}(c), \cdots, u_{n}(c)\right)$ again form a module $N$, if $u$ varies over $F$ for fixed $(c)$. Let $\left(y_{1}{ }^{1}, y_{2}{ }^{1}, \cdots, y_{n}^{1}\right),\left(y_{1}{ }^{2}, \cdots, y_{n}^{2}\right)$, $\cdots,\left(y_{1}{ }^{s}, \cdots, y_{n}{ }^{8}\right)$ form a basis of $N,(s \leqq n)$. Without restriction of generality we may assume that this basis forms an orthogonal system:

$$
\sum_{l=1}^{n} y_{l}^{j} y_{l}^{k}=\delta_{i k}, \quad i, k=1, \cdots, s .
$$

* Cf. L. L. Dines, this Bulletin, vol. 42, p. 357, and the paper of R. W. Stokes Transactions of this Society, vol. 33, p. 782 et seq.

$\dagger$ Bonnesen-Fenchel, Theorie der Konvexen Körper, p. 9. 
Let $u^{1}(x, c), u^{2}(x, c), \cdots, u^{s}(x, c)$ be the functions of $F$ corresponding to the vectors

$$
u_{i}^{k}(c, c)=y_{i}^{k}
$$

Then for every $u$ in $F$

$$
u_{i}(c)=\sum_{j=1}^{s} \lambda_{j} y_{i}^{j}
$$

with

$$
\lambda_{j}=\sum_{k=1}^{n} u_{k}(c) y_{k}^{j}
$$

Thus

$$
u_{i}(c)=\sum_{j=1}^{s} \sum_{k=1}^{n} u_{k}(c) u_{k}^{j}(c, c) u_{i}^{j}(c, c) .
$$

Consider the function

$$
\bar{u}(x)=u(x)-\sum_{j=1}^{s} \sum_{k=1}^{n} u_{k}(c) u_{k}^{j}(c, c) u^{j}(x, c) .
$$

Then $\bar{u}$ is in $F$. We have

$$
\bar{u}_{i}(c)=u_{i}(c)-\sum_{j=1}^{s} \sum_{k=1}^{n} u_{k}(c) u_{k}^{j}(c, c) u_{i}^{j}(c, c)=0 .
$$

Hence $\bar{u}$ is in $\phi$. Consequently

$$
0=\sum_{i, k} a_{i h}(c) \bar{u}_{i h}(c)=\sum_{i, h} a_{i h}(c) u_{i h}(c)-\sum_{i, j, k, h} a_{i h}(c) u_{k}(c) u_{k}^{j}(c, c) u_{i h}^{j}(c, c) .
$$

Thus $u(x)$ satisfies the elliptic equation

$$
0=\sum_{i, h} a_{i h}(x) u_{i h}(x)-\sum_{i, j, k, h} a_{i h}(x) u_{k}^{j}(x, x) u_{i h}^{j}(x, x) u_{k}(x) .
$$

UNIVERSITY OF KENTUCKY 